\title{
COMÉRCIO JUSTO: EM QUE ESTÁGIO ESTAMOS?
}

\author{
Marilia Bonzanini Bossle \\ Doutoranda em Administração pela Universidade Federal do Rio Grande do Sul - UFRGS \\ mariliabossle@gmail.com
}

\section{Eugênio Ávila Pedrozo}

Doutor em Genie Industrielle pela Institut National Polytechnique de Lorraine

Professor da Universidade Federal do Rio Grande do Sul - UFRGS

eapedrozo@ea.ufrgs.br

\section{RESUMO}

O comércio justo, mesmo se tratando de um tema de pesquisa recente, tem ganhado ênfase crescente na agenda de pesquisa. Identificam-se discussões e publicações sobre o tema em eventos, mas nem sempre baseados em uma literatura qualificada. Portanto, já se impõe a necessidade de se compreender a trajetória e fazer um mapeamento para verificar o estado da arte. Nesse sentido, neste artigo, busca-se fazer uma revisão da literatura sobre os artigos da área temática de comércio justo. Esse objetivo permite uma contribuição para avançar no uso de artigos qualificados sobre o tema, além do resultado em si do estado da arte. Optou-se pelo estudo bibliométrico para se obter uma visão geral e identificar a influência de diferentes revistas. Mesmo que a ideia principal não seja a de ranquear os periódicos, os índices e fatores de impactos principais utilizados nas bases de dados foram verificados, focando-se na sua relevância para a área. As bases de dados analisadas foram: Ebsco, ISI e Scopus. Foram identificados autores e suas origens acadêmica e institucional, a fim de identificar a existência de Centros de Referência no assunto. Os resultados destacam Reino Unido e Estados Unidos como as principais regiões envolvidas na produção científica sobre comércio justo. A autora com mais artigos e a universidade em destaque são americanas. A Revista que mais se destacou foi a Journal of Business Ethics. Observou-se também que mesmo que poucas revistas tenham classificação no qualis, elas apresentam bons indicadores de impacto, sendo consideradas relevantes para a pesquisa acadêmica em administração.

Palavras-chave: Bases de dados; Comércio Justo; Estudos bibliométricos; Fatores de impacto; Revistas científicas.

\section{ABSTRACT}

\section{FAIR TRADE: AT WHICH STAGE ARE WE?}

Fair Trade, despite being a subject of recent research, has gained increasing emphasis on the research agenda. It is possible to identify publications and discussions about the topic on events, although not always based on a qualified literature. Therefore, it is necessary to understand the trajectory and make a map to check the state of the art in this issue. Accordingly, this paper aims to do a literature review of articles on the subject area of Fair Trade. This goal allows a contribution to advancing the use of qualified articles on the subject, beyond the result itself of the state of the art. We chose the bibliometric study to get an overview and to identify the influence of different journals. Even if the main idea is not to rank journals, impact factors were checked in order to know its relevance to the analyzed area. We searched the following databases: Ebsco, ISI and Scopus. In the sample were identified authors and their academic background and institutional framework in order to identify the existence of referral centers in the topic. The results highlight the United Kingdom and the United States as the main regions involved in the scientific literature on Fair Trade. The author and the university with more articles featured are American. The journal that stood out was the Journal of Business Ethics. It was also observed that although few journals have classification in qualis, they have good indicators of impact, and may be considered relevant to academic research in management.

Key words: Bibliometric studies; Databases; Fair trade; Impact factors; Journals. 


\section{INTRODUÇÃO}

Conhecer os autores e campos de estudo da área de interesse ajuda a delinear um determinado campo de atuação e, consequentemente, a trajetória dos autores interessados no assunto. Dessa forma e considerando a importância da revisão da literatura e de estudos bibliométricos para a avaliação e identificação da influência de diferentes revistas e visões sobre determinado assunto, optou-se por fazer um estudo bibliométrico sobre comércio justo.

Holden, Rosenberg, Barker e Lioi (2010) afirmam que no campo social é muito importante que se veja a trajetória que os artigos irão seguir e como são as publicações empíricas. Neste aspecto, a revisão da literatura e estudos bibliométricos contribuem para avaliar a influência de diferentes revistas, para ver o impacto científico, para obter a visão geral de um determinado campo (Bakker, Groenewegen, \& Hond, 2005; Meho \& Yang, 2007). Muitos estudos bibliométricos são realizados para conhecer uma determinada área, seus autores, campos de estudos, entre outros.

O comércio justo é um conceito novo, mas que já está despertando o interesse de pesquisadores, empresas, organizações não-governamentais e do público em geral. O comércio justo visa pagar melhores preços aos produtores, proporcionando condições de trabalho decentes e adequadas, e com critérios justos nas relações entre empregadores e empregados, principalmente nos países em desenvolvimento. Por meio da melhor remuneração aos produtos, o comércio justo foca no melhoramento das condições dos produtores, para que estes alcancem melhores condições e para que possam ter poder sobre suas próprias vidas, sem depender de terceiros. O objetivo é acabar com a pobreza e fortalecer as famílias e os lugares onde vivem (Fairtrade Foundation, 2010; Max Havellar, 2010).

Com relação à disseminação do comércio justo, acredita-se que uma divulgação mais ampla dos conceitos, com apoio da sociedade, empresas, universidade e governo poderia ajudar. Destacase, nesse caso, o papel da academia e sua forma principal para a difusão do conhecimento, por meio das publicações científicas. Nesse sentido, destaca-se o uso de estudos bibliométricos.

O objetivo deste trabalho é o de fazer uma revisão da literatura sobre os artigos da área temática de comércio justo. Por meio desse mapeamento procura-se identificar as regularidades, os autores, as universidades ou os centros de referência e as revistas científicas para publicação. Nesse sentido, identificam-se os artigos e periódicos da área, procurando uma tendência e indicações sobre os melhores periódicos para publicação, com vistas a auxiliar e direcionar quem está estudando o tema ou pretende começar a fazê-lo. A ideia principal não é ranquear os periódicos, mas é necessário buscar sua classificação, com vistas a conhecer a sua relevância na área. Dessa forma, os índices e fatores de impactos principais utilizados nas bases de dados foram verificados.

É relevante citar também que se trata de um tema mundialmente novo. O Brasil ainda está iniciando sua agenda de pesquisa em Comércio Justo, tanto que o artigo mais antigo encontrado nas bases de dados consta do ano de 1990.

Este artigo é composto desta introdução, de uma breve revisão da literatura sobre o conceito de comércio justo, estudos bibliométricos, os principais índices de referência e fatores de impacto. Na sequência está descrito o método utilizado para a construção do estudo, seguido dos resultados e das considerações finais.

\section{COMÉRCIO JUSTO}

Entende-se por comércio justo, a associação comercial orientada para o desenvolvimento sustentável e para os produtores excluídos ou em desvantagem, propondo melhores condições comerciais, onde se paga um preço justo para os produtores. Permite-se, dessa maneira, uma melhoria substancial na qualidade de vida dos produtores e consumidores. O consumidor consciente não adquire apenas produtos, mas estabelece também relações de compromisso com os produtores. Ele faz questão de estar informado sobre a origem dos produtos, seu conteúdo ético e ambiental. Assim, as técnicas de produção devem ser favoráveis ao meio ambiente e a organização da 
produção deve ser democraticamente administrada (Fretel \& Simoncelli-Bourque, 2003; Mcmurtry, 2009).

A ampliação do acesso dos consumidores aos produtos do comércio justo permite que a base de clientes desse mercado fique maior e que se movimente de um círculo restrito de compra para um segmento mais numeroso de consumidores conscientes e preocupados com a situação (Gendron, Bisaillon, \& Rance, 2009).

Por isso, quando se fala em expandir e difundir um movimento social, deve-se pensar em formas coletivistas de divulgação para que a mudança desejada seja efetiva. As alternativas coletivistas são construídas em torno de conceitos como o de liderança ética, ou seja, com organizações que podem exercer o papel de lideranças, desenvolvendo relações afetivas para promover o comércio justo e ético. Para isso, é importante o desenvolvimento de espaços, que podem ser geográficos ou virtuais, mas a escolha por um produto ético deve ser a escolha dominante e não apenas uma entre muitas alternativas (Low \& Davenport, 2009).

\section{ESTUDOS BIBLIOMÉTRICOS}

Segundo Holden et al. (2010), no campo social, é muito importante que se veja a trajetória que os artigos irão seguir e como são as publicações empíricas. Os estudos bibliométricos são utilizados para obtenção de uma visão geral de um campo e são orientados para a busca de estruturas e padrões, por exemplo, autores-chave, revistas mais conceituadas e orientações epistemológicas (Bakker, Groenewegen, \& Hond, 2005).

Os autores Bakker et al. (2005) constataram que embora as pesquisas sobre Responsabilidade Social Corporativa (RSC) sejam feitas há um bom tempo, não há unanimidade quanto a evolução deste campo de estudo. Por isso resolveram fazer um estudo bibliométrico, pois queriam explorar esse processo evolutivo. Ainda assim, este artigo de Bakker et al. (2005) causou muita polêmica e eles tiveram que fazer outro como resposta às críticas recebidas (Bakker, Groenewegen, \& Hond, 2006).

Bakker et al. (2006) fizeram uma nova tabela com novas buscas e agregaram às tabelas novas bases de dados, Scholar Google e Scopus, que não existiam na época do outro artigo. Ainda assim, afirmam que é perigoso e míope usar a frequência de citação como o único indicador de qualidade acadêmica. Muitas das críticas vieram de autores que não quiseram ser deixados de fora. Bakker et al. (2006) se justificam dizendo que eles não tinham a intenção de fazer a lista completa da bibliografia sobre RSC, mas sim a de fazer um mapeamento sobre como esses conceitos eram estabelecidos ao longo do tempo e na busca por regularidades (Bakker et al., 2006).

A revisão da literatura e os estudos bibliométricos contribuem para avaliar a influência de diferentes revistas, para ver o impacto científico, para obter a visão geral de um determinado campo (Bakker et al., 2005; Meho \& Yang, 2007). Outra vantagem, é que pode sugerir como um determinado campo de estudo pode avançar. A pesquisa pode influenciar as empresas sobre qual a maneira mais eficiente de ela preencher suas reais e efetivas responsabilidades. Neste sentido, o que os pesquisadores e estudiosos têm a dizer é essencial para as empresas com relação aos riscos e problemas sociais que podem ser evitados. O significado prático da agenda de investigação não é, portanto, menos importante do que suas implicações teóricas (Margolis \& Walsh, 2003).

Os estudos bibliométricos também são importantes, pois por meio deles artigos duplicados, ou seja, aqueles publicados em duas revistas diferentes, podem ser identificados. Um estudo de Larivière e Gingras (2010), indicou que existem sim trabalhos iguais publicados em revistas diferentes e que estes, geralmente, são do mesmo ano ou com diferença de um ano. Isto sugere que foram submetidos simultaneamente para mais de uma revista. Os autores identificaram que grande parte desses casos acontece na área médica e um pequeno número na área de ciências sociais (Larivière \& Gingras, 2010).

Uma técnica chave nas análises bibliométricas, é a análise de citação (Holden et al., 2010). Este tipo de análise foca na avaliação quantitativa e em padrões de citação e estes padrões são 
diferentes entre uma base de dados e outra. Por isso, é discutível se esta análise é um bom indicador sobre a relevância ou não de determinado autor (Holden et al., 2010).

Com relação ao fator de impacto, também existem diferentes maneiras de calculá-lo e cada base de dados pode escolher a maneira que quiser. $O$ fator de impacto pode ser usado em bibliotecas, para escolher os journals mais relevantes ou mesmo para a avaliação de departamentos, instituições e até de pesquisadores (Cameron, 2005). Porém, existem críticas ao uso de fator de impacto. Segundo Cameron (2005) há fortes indícios de que os editores podem manipular os fatores de impacto.

Mesmo que haja alguns problemas, é importante que tenhamos indexadores para avaliação. $\mathrm{O}$ controle de citações é feito cruzando as referências dos artigos. Isso pode ser mais eficaz de se fazer em uma época como a de hoje, que há uma grande quantidade de revistas acadêmicas, artigos, além da constante multidisciplinariedade presente nos trabalhos acadêmicos (Cameron, 2005).

As deficiências dos fatores de impacto da ISI, identificados por Cameron (2005), são: i) a janela de dois anos para a avaliação das citações - esses dois anos são um bom indicativo, mas não estão livres de falhas; ii) números de revistas publicados naquela área - quanto maior o número de revistas sobre determinado tema, maior o fator de impacto geral; iii) a cobertura do ISI - não tem todas as revistas; iv) idioma.

Então, o fator de impacto mede a citação, mas não necessariamente a importância do artigo. A avaliação bibliométrica de artigos publicados tende a ignorar o uso que é feito pelo conhecimento gerado pelas conclusões de determinados artigos. Por exemplo, na área da medicina, artigos são lidos para melhorar os diagnósticos e salvar vidas. As publicações em uma revista com alto valor de impacto não vai, portanto, significar que o artigo será altamente citado, influente e de alta qualidade (Cameron, 2005). Mesmo assim, existe a vantagem de publicar nessas revistas, pois mesmo que o artigo não seja muito citado, é provável que ele tenha maior visibilidade.

Usar o método de avaliação da demanda por determinado artigo, por meio da contagem de citação, apresenta uma falha significativa: os artigos mais antigos têm mais chance de serem citados. Com o aumento do número de revistas, a oportunidade de ser citado muda ao longo do tempo. Uma maneira de amenizar essa falha é comparar os resultados anuais de citação com o número médio de citações do artigo publicados na revista do ano em observação (Walsh, Weber, \& Margolis, 2003).

\section{1 Índices e fatores de impacto}

Mesmo que existam críticas aos fatores de impacto, é necessário o estabelecimento de parâmetros para a classificação dos periódicos e revistas acadêmicas. Conforme visto, Bakker et al. (2006) e Holden et al. (2010) consideram que a análise de citação não deve ser usada isoladamente, assim como Cameron (2005) lista uma série de deficiências dos indexadores. Ainda assim, Cameron (2005) afirma que mesmo que haja alguns problemas, é importante que tenhamos índices para avaliação.

Como as bases de dados utilizam diferentes recursos para avaliar as suas revistas, nesse artigo são apresentados os seguintes índices utilizados pelas bases de dados pesquisados: Journal Citation Reports (JCR); Índice H; Source Normalized Impact per Paper (SNIP); SCImago Journal Rank (SJR). Além disso, quando estiver classificado no Qualis da área de Administração, Ciências Contábeis e Turismo será indicado.

A ISI of Knowledge mede o fator de impacto das revistas por meio do Journal Citation Reports (JCR), que é um tipo de medida de fator impacto baseado nos dados de citação. Esse índice foi desenvolvido por Eugene Garfield no Institute for Scientific Information (ISI), atualmente Thomson Reuters e é pioneiro nesse ramo e um dos mais amplamente utilizados para estudos bibliométricos (Moed, 2009).

$\mathrm{O}$ índice $\mathrm{H}$ foi proposto por Hirsch e inicialmente era utilizado apenas para medir a produção dos cientistas, mas atualmente é utilizado também para medir o impacto das revistas, se 
baseando na mesma forma de cálculo. Esse indicador se baseia na lista de publicações ranqueadas em ordem decrescente ordenada pelo número de citações. $\mathrm{O}$ valor de $\mathrm{H}$ será o número de artigos (N) na lista que tiverem $\mathrm{N}$ ou mais citações (Hirsch, 2005).

A base Scopus utiliza também duas outras medidas para verificar o desempenho das revistas. Segundo eles, a justificativa para se usar as duas métricas está baseada no fato de não existirem ferramentas únicas e absolutas para medir o impacto, assim, usando duas formas de medição é possível ter um resultado mais acurado. As duas medidas são o Source Normalized Impact per Paper (SNIP) e o SCImago Journal Rank (SJR).

Desenvolvido pelo professor Henk Moed, do Centro de Estudos de Ciência e Tecnologia (CWTS - do inglês Centre for Science and Technology Studies), o SNIP, mede o fator de impacto contextual de um periódico. Ele permite a comparação direta entre periódicos de diferentes campos disciplinares, considerando a frequência com que autores citam outros artigos, a velocidade de maturação do fator de impacto, e a extensão em que a base de dados abrange a literatura da área. Esse índice faz uma normalização dos valores de citação, levando em conta a frequência de citações de determinado campo de estudo. O SNIP é definido como a relação da contagem de citação do periódico por artigo e o potencial de citação em seu campo de assunto. Essa forma objetiva permitir a comparação direta entre fontes de diferentes campos de estudos (Moed, 2009).

O SCImago Journal Rank (SJR) foi desenvolvido pelo professor Félix Moya e é baseado na ideia de que nem todas as citações foram criadas da mesma maneira. Assim, o campo de estudo, a qualidade e reputação da revista tem um impacto direto no valor da citação. Isso quer dizer que uma citação de fonte com um alto SJR cria um maior valor do que a citação de uma fonte com baixo SJR.

Qualis é o conjunto de procedimentos utilizados pela Coordenação de Aperfeiçoamento de Pessoal de Nível Superior [Capes] para estratificação da qualidade da produção intelectual dos programas de pós-graduação brasileiros. A estratificação da qualidade dessa produção é realizada de forma indireta. Dessa forma, o Qualis afere a qualidade dos artigos e de outros tipos de produção, a partir da análise da qualidade dos veículos de divulgação, ou seja, periódicos científicos (Coordenação de Aperfeiçoamento de Pessoal de Nível Superior [CAPES], 2011).

A classificação de periódicos é realizada pelas áreas de avaliação e passa por processo anual de atualização. Esses veículos são enquadrados em estratos indicativos da qualidade - A1, o mais elevado; A2; B1; B2; B3; B4; B5; até C que tem peso zero. Note-se que o mesmo periódico, ao ser classificado em duas ou mais áreas distintas, pode receber diferentes avaliações. Isto não constitui inconsistência, mas expressa o valor atribuído, em cada área, à pertinência do conteúdo veiculado (CAPES, 2011). Aqui nesse artigo são consideradas as avaliações realizadas pela área de Administração, Ciências Contábeis e Turismo.

\section{MÉTODO DE PESQUISA}

Segundo Meho e Yang (2007), utilizar a base de dados Institute for Scientific Information's (ISI) ou também chamada de Web of Science tem uma boa cobertura para journals. Dessa forma, por meio da revisão da literatura e de conversas com pesquisadores da área, decidiu-se usar as três bases de dados consideradas as mais relevantes e completas em Ciências Sociais Aplicadas, mais especificamente, em Administração. As bases pesquisadas foram: Ebsco, ISI e Scopus. Foram considerados artigos com seis ou mais páginas. Esse critério foi adotado, pois esse é o número de páginas mínimo exigido pelas revistas científicas da área. Foram excluídos também os artigos referentes à lei americana, a Fair Trade Law, por não ter relação direta com o conceito de interesse na pesquisa.

A pesquisa na ISI foi realizada no dia 12 de março de 2010. A busca foi feita com a expressão "Fair Trade" no título (title), pois não havia a opção de palavra chave (keyword). Foram encontrados 493 resultados. Deste resultado, foi feito um refinamento da pesquisa apenas para artigos e ficaram 242. Foram excluídos 117 artigos por falta de adequação de forma ou conteúdo. 
A pesquisa na base EBSCO, foi feita no dia 15 de março de 2010, primeiramente com a expressão "Fair Trade" no título, quando apareceram 175 artigos. Em seguida, foi feita a busca com a mesma expressão, mas em todo o texto (All text), com restrições a revistas e reviews acadêmicos. Foram encontrados 352 artigos, sendo que na primeira seleção, "Academic Journal", 119 artigos; na segunda, "Periodical", 136; e, na terceira, "Periodical", 46 artigos. Foram excluídos os "journal article" e "Trade Publications" por serem considerados menos importantes na comparação com as publicações científicas. Além destes, foram excluídos 192 artigos por não se adequarem à pesquisa devido ao número insuficiente de páginas, fuga do tema ou falta de alguma informação importante como autor, ano de publicação, etc.

A pesquisa na base de dados Scopus foi realizada no dia 13 de abril de 2010. A busca foi com a expressão "Fair Trade" nos campos title, ou keyword, onde foram encontrados 335 resultados. Foram excluídos os conference paper, editorial, letter. Ficaram article, review, short survey, article in press, que totalizaram 293 artigos. Destes, 72 foram excluídos por fugirem do tema ou por não terem o número de páginas suficiente.

Tabela 1: Amostra total de artigos sobre comércio justo

\begin{tabular}{|c|c|c|c|c|}
\hline Base & $\begin{array}{c}\text { Artigos } \\
\text { encontrados }\end{array}$ & Artigos excluídos & Repetidos & Amostra total \\
\hline EBSCO & 352 & 192 & & \\
\hline ISI & 242 & 117 & 238 & $\mathbf{2 6 8}$ \\
\hline Scopus & 293 & 72 & & \\
\hline
\end{tabular}

Fonte: dados da pesquisa

Conforme a tabela 1, após as buscas e exclusões de artigos por inadequação ao tema, insuficiente número de páginas ou de outras informações importantes, como por exemplo, autoria, foram feitos os cortes por repetição. Muitos artigos apareciam em mais de uma base de dados, no total, foram 238 repetições. Após tudo isso, chegou-se a amostra final, com 268 artigos. Nestes, foi feita a análise que será apresentada a seguir.

Nestes 268 artigos, verificaram-se quantos autores tinham por artigo. Foram 454 respostas, sendo 368 diferentes autores. Destes, 51 autores apareceram mais de uma vez em 137 citações. A fim de verificar a existência de Centros de Referência com relação ao assunto, foram pesquisadas a origem acadêmica destes autores, ou seja, foram observadas suas universidades, filiação institucional e países de origem. Adicionalmente, foram listadas as revistas que apareceram com mais frequência e verificados os seus índices de fatores de impacto.

\section{ANÁLISE E DISCUSSÃO DOS RESULTADOS}

Foram encontrados 268 artigos em 158 revistas e 368 autores diferentes. A análise dos autores, universidades e seus respectivos países de origem foram realizadas com os 51 autores mais prevalentes, que tiveram 137 aparições. A análise será feita com os autores e países mais prevalentes, seguidos das revistas e das universidades, a fim de localizar a existência de centros de referência na área.

\subsection{Autores e países}

Considerando os 51 autores mais prevalentes, ou seja, aqueles que publicaram mais de um artigo, foram verificados quais os países de origem da ligação acadêmica de cada autor. Como se observa na tabela 2, os Estados Unidos (EUA) e o Reino Unido são os países mais prevalentes, seguidos de Canadá, Brasil, França, Bélgica, China, Coreia do Sul, Finlândia, Holanda, Itália, México e Nova Zelândia.

Como esses autores publicaram mais de um artigo e muitos artigos têm mais de um autor, ao fazer a relação de quantos artigos há por autor e por país, foram encontradas 137 citações. Por meio 
dessas tabelas, pode-se perceber que os Estados Unidos e Inglaterra são os países relevantes em publicações sobre comércio justo. O Canadá aparece em terceiro lugar, mas também se destaca pelo volume de publicações.

Tabela 2: Países dos 51 autores analisados

\begin{tabular}{lr}
\hline País & Frequência \\
\hline EUA & $\mathbf{1 8}$ \\
\hline Reino Unido & $\mathbf{1 6}$ \\
Canada & 5 \\
Brasil & 2 \\
França & 2 \\
Bélgica & 1 \\
China & 1 \\
Coreia do Sul & 1 \\
Finlândia & 1 \\
\hline Holanda & 1 \\
Itália & 1 \\
México & 1 \\
Nova Zelândia & 1 \\
\hline Total & 51 \\
\hline
\end{tabular}

Fonte: dados da pesquisa

Tabela 3: Países das 137 citações

\begin{tabular}{lr}
\hline País & Frequência \\
\hline EUA & $\mathbf{5 2}$ \\
Reino Unido & $\mathbf{4 0}$ \\
Canadá & $\mathbf{1 7}$ \\
França & 4 \\
Brasil & 4 \\
Itália & 5 \\
Bélgica & 2 \\
Nova Zelândia & 3 \\
Holanda & 2 \\
China & 2 \\
Coreia do Sul & 2 \\
Finlândia & 2 \\
México & 2 \\
\hline TOTAL & 137 \\
\hline
\end{tabular}

Fonte: dados da pesquisa

Conforme as informações das tabelas 2 e 3, é possível observar que, apesar de ter sido um local pioneiro para o comércio justo e sua consolidação por meio do início da certificação em 1988 (Fretel \& Simoncelli-Bourque, 2003), a Holanda aparece com apenas dois artigos publicados. Estas publicações são de um único autor cuja base institucional é a Holanda.

\subsection{Autores, universidades e centros de referência}

Com relação aos autores, a figura 1 apresenta todos os autores que possuem mais de um artigo publicado sobre o tema. Há um autor que se destaca com nove artigos publicados. Esta autora, Laura Raynolds, é professora do Departamento de Sociologia da Universidade do Colorado, nos Estados Unidos e também codiretora do Centro de Estudos para um Comércio Alternativo e 
Justo. Como veremos adiante, estas duas instituições são consideradas centros de referência para estudos na área.

\begin{tabular}{|c|c|}
\hline Autores & No de Artigos \\
\hline Raynolds, L.T. & 9 \\
\hline Becchetti, L. & 5 \\
\hline Murray, D.L. & 5 \\
\hline Davies, I.A. & 4 \\
\hline Fridell G & 4 \\
\hline Goodman, M.K. & 4 \\
\hline Hudson, I. & 4 \\
\hline Hudson, M. & 4 \\
\hline Littrell, M.A. & 4 \\
\hline Tallontire, A. & 4 \\
\hline Taylor, P.L. & 4 \\
\hline Bacon, C.M. & 3 \\
\hline Davenport, E. & 3 \\
\hline Doherty, B. & 3 \\
\hline Halepete, J. & 3 \\
\hline Low, W. & 3 \\
\hline Moore, G. & 3 \\
\hline Abdelgawad, W. & 2 \\
\hline Arnould, E.J. & 2 \\
\hline Audebrand LK & 2 \\
\hline Ball, D. & 2 \\
\hline Bhagwati, J. & 2 \\
\hline Biggs, S. & 2 \\
\hline Burgoon, B. & 2 \\
\hline Cavalett, 0. & 2 \\
\hline De Pelsmacker, P. & 2 \\
\hline Dine, J. & 2 \\
\hline Doran, C.J. & 2 \\
\hline Fisher, E. & 2 \\
\hline Golding, K.M. & 2 \\
\hline Hayes, M.G. & 2 \\
\hline Howard, P.H. & 2 \\
\hline Jaffee, D. & 2 \\
\hline Le Mare, A. & 2 \\
\hline Le Velly, R. & 2 \\
\hline Linton, A. & 2 \\
\hline Lyon, S. & 2 \\
\hline Nicholls, A. & 2 \\
\hline Ortega, E. & 2 \\
\hline Park, J. & 2 \\
\hline Plastina, A. & 2 \\
\hline Renard, M.C. & 2 \\
\hline
\end{tabular}




\begin{tabular}{|c|c|}
\hline Rice, R.A. & 2 \\
\hline Shreck, A. & 2 \\
\hline Smith, A.M. & 2 \\
\hline Utting, K. & 2 \\
\hline Valkila, J. & 2 \\
\hline Varul, M.Z. & 2 \\
\hline Watson, M. & 2 \\
\hline Weber, J. & 2 \\
\hline Welford, R. & 2 \\
\hline
\end{tabular}

Figura 1: Autores mais prevalentes

Fonte: dados da pesquisa

Notadamente a Universidade do Colorado (Colorado State University, 2010) é a mais citada, com 22 observações. Destaca-se que dos 51 autores, quatro são dessa Universidade e todos com um número significativo de publicações em comércio justo: Littrell, M.A., com quatro artigos; Murray, D.L., com cinco; Raynolds, L.T., com nove; e, Taylor, P.L., com quatro.

A Universidade do Colorado destaca-se como um Centro de Referência não só por esses pesquisadores, mas também por ter o Centro de Estudos para um Comércio Alternativo e Justo (do inglês Center For Fair \& Alternative Trade, CFAT), ligado à Universidade do Colorado. Além disso, nesta universidade, tem um grupo de pesquisa em comércio justo - Fair Trade Research Group. Os quatro autores estão envolvidos nestes grupos e centro de referência.

O CFAT é um centro de pesquisa que se empenha para o crescimento do mercado baseado em mudança social e proteção ambiental. Por meio de pesquisas e atividades de campo, o CFAT faz análises das iniciativas em andamento, de futuras oportunidades e dilemas em curso para entender melhor o potencial e os limites do comércio justo, dos sistemas de certificação e outros aspectos que emergem do movimento do consumo consciente. Suas principais atividades são: i) Pesquisa acadêmica; ii) Treinamento de graduados e pós-graduados de Mestrado e Doutorado; iii) Consultorias para o setor público e privado; iv) Workshops, conferências e simpósios; e, assistência técnica para grupos, produtores, Organizações Não-Governamentais, entre outros (Center For Fair \& Alternative Trade, 2010).

Quem aparece em segundo lugar na frequência de artigos publicados por universidade, é a Universidade de Manitoba, no Canadá, com oito referências. Apesar de o Reino Unido ser o segundo na frequência de publicações na área, suas publicações são de pesquisadores de diferentes universidades, com destaque à Universidade de Leeds (University of Leeds), com seis referências e a King's College London, com quatro.

\subsection{Revistas para publicação}

Conforme se observa na tabela 4 , de todos os 268 artigos publicados, foram identificadas 158 revistas diferentes, mas apareceram apenas 18 revistas com três ou mais artigos publicados. Entre elas, destaca-se a revista Journal of Business Ethics, com vinte e cinco artigos publicados, seguidos da Sustainable Development, com dez e da Agriculture and Human Values, com oito. Foram verificados também os seguintes fatores de impacto: Journal Citation Reports (JCR); Índice $\mathrm{H}$; Source Normalized Impact per Paper (SNIP); SCImago Journal Rank (SJR). Foi indicada também a classificação da revista, quando esta estiver no Qualis da área de Administração, Ciências Contábeis e Turismo.

Tabela 4: Revistas e fatores de impacto 
Comércio justo: em que estágio estamos?

\begin{tabular}{|c|c|c|c|c|c|c|}
\hline Revistas & Frequência & JCR 2009 & $\begin{array}{c}\text { Índice } \\
\text { H }\end{array}$ & $\begin{array}{l}\text { SNIP } \\
2009\end{array}$ & $\begin{array}{l}\text { SJR } \\
2009\end{array}$ & $\begin{array}{l}\text { Qualis } \\
\text { para } \\
\text { ADM }\end{array}$ \\
\hline Journal Of Business Ethics & 25 & 1,088 & 45 & 0,739 & 0,043 & A1 \\
\hline Sustainable Development & 10 & 1,104 & 17 & 0,551 & 0,047 & não tem \\
\hline Agriculture and Human Values & 8 & 1,123 & 25 & 0,565 & 0,054 & não tem \\
\hline Development in Practice & 7 & -------------- & 14 & 0,174 & 0,037 & não tem \\
\hline World Economy & 7 & 1,159 & 24 & 0,715 & 0,042 & não tem \\
\hline World Development & 6 & 1,225 & 65 & 1,119 & 0,07 & A1 \\
\hline Journal of International Development & 5 & -------------- & 25 & 0,258 & 0,062 & A2 \\
\hline Geoforum & 4 & 1,574 & 40 & 0,575 & 0,054 & não tem \\
\hline $\begin{array}{l}\text { Globalizations } \\
\end{array}$ & 4 & \multicolumn{5}{|c|}{ Sem informação } \\
\hline Journal of Consumer Policy & 4 & -------------- & 16 & 0,219 & 0,037 & não tem \\
\hline Journal of Rural Studies & 4 & 2,348 & 39 & 1,253 & 0,054 & não tem \\
\hline Small Enterprise Development & 4 & \multicolumn{5}{|c|}{ Sem informação } \\
\hline $\begin{array}{c}\text { Clothing and Textiles Research } \\
\text { Journal }\end{array}$ & 3 & & 11 & 0,474 & 0,038 & não tem \\
\hline Development & 3 & 7,194 & 205 & 1,307 & 2,538 & não tem \\
\hline Economic Affairs & 3 & -------------- & 7 & 0,067 & 0,035 & não tem \\
\hline Forests Trees and Livelihoods & 3 & --------------- & 11 & 0,275 & 0,04 & não tem \\
\hline $\begin{array}{c}\text { International Journal of Tourism } \\
\text { Research }\end{array}$ & 3 & -------- & 3 & 0,208 & 0,036 & não tem \\
\hline $\begin{array}{l}\text { Journal of Agricultural and Food } \\
\text { Industrial Organization }\end{array}$ & 3 & -------------- & 6 & 0,484 & 0,056 & não tem \\
\hline
\end{tabular}

Fonte: dados da pesquisa

Conforme a tabela 4, a revista Journal of Business Ethics é a com o maior número de publicações sobre o comércio justo. É pertinente notar que esta é uma revista A1, conforme a avaliação Qualis Capes (CAPES, 2010) e segundo seu editorial, aceita artigos originais com uma ampla variedade, tanto metodológica quanto disciplinar, que tragam algo novo ou único em relação às discussões sobre o assunto tratado (Springer, 2010).

Várias dessas revistas ainda não estão classificadas no Qualis da área de administração. Isso se justifica pelo fato de que é um tema novo e pouco estudado por pesquisadores brasileiros e as revistas só são classificadas depois de algum pesquisador ligado a instituições da área já ter publicado. Mesmo assim, nota-se que essas revistas apresentam um fator de impacto significativo para a área. Segundo o documento da área, um artigo com fator de impacto JCR maior que 0,5 pode ser considerado A1, menores que 0,5 seriam A2. Para as revistas que não estão classificadas no JCR ou índice $\mathrm{H}$, são utilizados outros critérios para classificá-las, conforme está detalhado no documento de área da Capes. Para a área de Administração, Contabilidade e Turismo, um índice $\mathrm{H}$ maior que 5 é considerado significativo.

\section{CONSIDERAÇÕES FINAIS}

Salienta-se a importância de um estudo como esse para o comércio justo ao mostrar onde estão e quem são os principais pesquisadores sobre o tema. Os autores Bakker, Groenewegen e Hond (2005) fizeram um estudo bibliométrico sobre Responsabilidade Social Corporativa, pois constataram que não havia unanimidade quanto à evolução deste campo de estudo.

Low e Davenport (2009) afirmam a importância da criação de espaços éticos para a difusão e expansão do movimento do comércio justo. Nesse sentido, pode-se afirmar que o levantamento de artigos sobre o tema e um estudo bibliométrico podem ajudar a apontar onde poderiam ser desenvolvidos esses espaços e quais seriam as pessoas que apoiariam a iniciativa.

Atingindo seu objetivo inicial, o trabalho mapeou os artigos da área de comércio justo para identificar por meio deles: regularidades, autores, universidades ou centros de referência e revistas científicas para publicação. Corroborando com a análise sobre a importância dos estudos 
bibliométricos para avaliar as diferentes revistas, viu-se nesse estudo uma concentração de artigos na revista Journal of Business Ethics. Dessa forma, foi disponibilizada aos pesquisadores uma lista de revistas para publicação de artigos relacionados.

As citações e os autores mais prevalentes nos artigos das bases de dados estudadas permitiram a identificação de alguns autores principais. Laura Raynolds se destaca como autora com mais artigos publicados. Ela é pesquisadora de uma Universidade do Colorado, confirmando a importância dos Estados Unidos neste tipo de pesquisa. Raynolds é a diretora do Centro de Estudos para um Comércio Alternativo e Justo, também um centro de referência, destacando o papel dos EUA na pesquisa em comércio justo. A Universidade do Colorado (Colorado State University) é também a mais citada, com 22 observações.

Os Estados Unidos e o Reino Unido são os países mais prevalentes, os que mais apareciam como base institucional dos autores. Ainda que a pesquisa tenha sido feita predominantemente em inglês, não se pode negar a influência dessas duas regiões no estudo desse campo. Também foram feitas buscas em português e francês, mas para essas bases de dados, o resultado das buscas não teve relevância. $\mathrm{O}$ fato de a pesquisa ter sido feita em inglês pode ser uma limitação desse artigo.

Por ser um campo de estudo relativamente novo, não foram encontradas as revistas mais prevalentes no Qualis da Capes. Mas, isso se justifica também pelas limitações da análise de citações quantitativas citadas por Walsh, Weber e Margolis (2003). Segundo estes autores, uma das falhas de se medir a demanda de determinado artigo por análise de citações é que os artigos mais antigos tendem a apresentar um número maior de citações.

Neste artigo, foi pesquisada a origem acadêmica dos autores que apareceram mais de uma vez, foram 51 autores em 137 aparições nos artigos, com o intuito de verificar a existência de Centros de Referência com relação ao estudo sobre Comércio Justo. Foram observadas as universidades e os países de origem. Como sugestão de pesquisas futuras, seria interessante fazer isso com todos os autores da amostra, para ampliar o conhecimento sobre centros de pesquisas e universidades com linhas de investigação relacionadas ao assunto.

Ainda para pesquisas futuras, outra ideia é investigar quais são as áreas e produtos do comércio justo que estão sendo estudados (frutas, artesanato, turismo, etc) e identificar quais são os métodos mais utilizados. Para a identificação de mais centros de pesquisa sobre comércio justo, pode-se também fazer a pesquisa em bases de dados de outros idiomas, tais como o francês e o português.

\section{REFERÊNCIAS}

Bakker, F. G. A., Groenewegen, P., \& Hond, F. (2005) A bibliometric analysis of 30 years of research and theory on corporate social responsibility and corporate social performance. Business Society, 44, 283-317.

Bakker, F. G. A., Groenewegen, P., \& Hond, F. (2006) A research note on the use of bibliometrics to review the corporate social responsibility and corporate social performance literature. Business Society, 45, 7-19.

Cameron, B. D. (2005). Trends in the usage of isi bibliometric data: uses, abuses, and implications. Libraries and the Academy, 5 (1), 105-125.

Coordenação de Aperfeiçoamento de Pessoal de Nível Superior (2010). WebQualis: consulta de periódicos. Recuperado em 10 junho, 2010, de http://qualis.capes.gov.br/webqualis/ConsultaPeriodicos.faces.

Coordenação de Aperfeiçoamento de Pessoal de Nível Superior (2011). Qualis Periódicos. Recuperado em 13 junho, 2011, de http://www.capes.gov.br/avaliacao/qualis. 
Center for Fair \& Alternative Trade (2010). About Us. Recuperado em 18 maio, 2010, de http://www.cfat.colostate.edu/about-us/.

Colorado State University (2010). Faculty Profile: Laura Raynolds. Recuperado em 25 abril, 2010, de http://www.colostate.edu/dept/sociology/faculty/laura.html.

Fairtrade Foundation (2010). Recuperado em 10 junho, 2010, de http://www.fairtrade.org.uk/what_is_fairtrade/faqs.aspx.

Fretel, A. C., \& Simoncelli-Bourque, E. (2003). O comércio justo e o consumo ético. Rio de Janeiro: DP\&A: Fase.

Gendron, C., Bisaillon, V., \& Rance, A. I. O. (2009). The institutionalization of fair trade: More than just a degraded form of social action. Journal of Business Ethics, 86 (1), 63-79.

Holden, G., Rosenberg, G., Barker, K., \& Lioi, J. (2010). Research on social work practice: a bibliometric evaluation of the first decade. Research on Social Work Practice, 20 (1), 11-20.

Larivière, V., \& Gingras, Y. (2010). On the prevalence and scientific impact of duplicate publications in different scientific fields (1980-2007). Journal of Documentation, 66 (2), 179-190.

Low, W., \& Davenport, E. (2009). Organizational leadership, ethics and the challenges of marketing fair and ethical trade. Journal of Business Ethics, 86 (1), 97-108.

Margolis, J. D., \& Walsh, J. P. (2003). Misery loves companies: rethinking social initiatives by business. Administrative Science Quarterly, 48, 268-305.

Max Havellar (2010). Fair trade. Recuperado em: 25 abril, 2010, de http://www.maxhavelaar.ch/en/maxhavelaar/fair-trade/.

Mcmurtry, J. J. (2009). Ethical value-added: fair trade and the case of café femenino. Journal of Business Ethics, 86, 27-49.

Meho, L. I., \& Yang, K. (2007). Impact of data sources on citation counts and rankings of lis faculty: web of science versus scopus and google scholar. Journal of the american society for information science and technology, 58 (13), 2105-2125.

Moed, H. F. (2009). Measuring contextual citation impact of scientific journals. Full documentation on SNIP: Version 13 November 2009. Recuperado em 9 junho, 2011, de http://arxiv.org/ftp/arxiv/papers/0911/0911.2632.pdf.

Springer (2010). Journal of Business Ethics. Recuperado em 10 junho, 2010, de http://www.springer.com/social+sciences/applied+ethics/journal/10551.

Walsh, J. P., Weber, K., \& Margolis, J. D. (2003). Social issues and management: our lost cause found. Journal of Management, 29 (6), 859-881.

Data do recebimento do artigo: 07/07/2011

Data do aceite de publicação: 20/08/2012

$\overline{\text { Revista de Gestão Social e Ambiental - RGSA, São Paulo, v. 6, n. 2, p. 33-44, maio/ago. } 2012 .}$ 